\title{
BioéthiqueOnline
}

\section{Les directives anticipées des personnes atteintes d'une démence de type Alzheimer : comment faire pour bien faire?}

\section{Line Perreault}

Volume 5, 2016

URI : https://id.erudit.org/iderudit/1044261ar

DOI : https://doi.org/10.7202/1044261ar

Aller au sommaire du numéro

Éditeur(s)

BioéthiqueOnline

ISSN

1923-2799 (numérique)

Découvrir la revue

Citer cet article

Perreault, L. (2016). Les directives anticipées des personnes atteintes d'une démence de type Alzheimer : comment faire pour bien faire? BioéthiqueOnline, 5. https://doi.org/10.7202/1044261ar
Résumé de l'article

La loi sur les soins de fin de vie est effective au Québec depuis quelques semaines. Cette loi prévoit la création d'un régime de directives médicales anticipées. Le respect des directives anticipées (DA) émises en prévision d'une inaptitude s'avère un moyen éthique bienfaisant d'assurer l'autodétermination du malade dans un contexte où l'inaptitude des personnes est totale et de courte durée (ex. : cancer en phase terminale). Mais qu'en est-il lorsque la perte des habiletés décisionnelles est graduelle et échelonnée sur une longue période de temps comme c'est le cas des personnes atteintes d'une démence de type Alzheimer (DTA)? Bien que la déchéance cognitive associée à la DTA rend légitime le recours aux DA pour favoriser l'autodétermination de ces personnes, se fier uniquement à des souhaits émis verbalement ou par écrit à ses proches ou à son médecin pour orienter les soins est une démarche apparaissant éthiquement risquée. Une révision de la littérature rattachée aux DA, l'étude des particularités cliniques associées à la DTA et l'analyse critique d’un cas clinique fictif, nous permettent d'énoncer la nécessité du développement d'une approche graduelle de revalidation des ententes qui tient compte des DA de la personne souffrant d'une DTA, tout en pondérant ses souhaits à son opinion en temps réel, sa qualité de vie actuelle et celle de son soignant. Le déploiement bienfaisant des DA repose sur la capacité des acteurs impliqués à considérer cette démarche comme un processus de revalidation des ententes. Ce changement nécessaire de paradigme assure que

l'actualisation de l'autodétermination des personnes atteintes d'une DTA ne se fasse pas au détriment de leur dignité, leur sécurité et leur intégrité. Une telle approche demeure à développer et à promouvoir pour les personnes atteintes d’une DTA au Québec. Des politiques de santé en ce sens et des repères normatifs spécifiques à cette clientèle doivent être rapidement mis de l'avant dans le contexte où le Québec associe le développement d'un régime de " directives médicales anticipées » à la Loi sur les soins de fin de vie.
Ce document est protégé par la loi sur le droit d'auteur. L’utilisation des services d’Érudit (y compris la reproduction) est assujettie à sa politique d'utilisation que vous pouvez consulter en ligne.

https://apropos.erudit.org/fr/usagers/politique-dutilisation/ 


\title{
Les directives anticipées des personnes atteintes d'une démence de type Alzheimer : Comment faire pour bien faire?
}

\author{
ARTICLE (RÉVISION PAR LES PAIRS / PEER-REVIEWED) \\ Line Perreault ${ }^{1}$ \\ Reçu/Received: 7 Feb 2015 \\ Publié/Published: 29 Jan 2016 \\ Éditeurs/Editors: Vanessa Chenel \& Charles Dupras \\ Évaluateurs externes/Peer-Reviewers: Alberto Bondolfi \& Christophe Trivalle
}

2016 L Perreault, Creative Commons Attribution 4.0 International License

\section{Résumé}

La loi sur les soins de fin de vie est effective au Québec depuis quelques semaines. Cette loi prévoit la création d'un régime de directives médicales anticipées. Le respect des directives anticipées (DA) émises en prévision d'une inaptitude s'avère un moyen éthique bienfaisant d'assurer l'autodétermination du malade dans un contexte où l'inaptitude des personnes est totale et de courte durée (ex. : cancer en phase terminale). Mais qu'en est-il lorsque la perte des habiletés décisionnelles est graduelle et échelonnée sur une longue période de temps comme c'est le cas des personnes atteintes d'une démence de type Alzheimer (DTA)? Bien que la déchéance cognitive associée à la DTA rend légitime le recours aux DA pour favoriser l'autodétermination de ces personnes, se fier uniquement à des souhaits émis verbalement ou par écrit à ses proches ou à son médecin pour orienter les soins est une démarche apparaissant éthiquement risquée. Une révision de la littérature rattachée aux DA, l'étude des particularités cliniques associées à la DTA et l'analyse critique d'un cas clinique fictif, nous permettent d'énoncer la nécessité du développement d'une approche graduelle de revalidation des ententes qui tient compte des DA de la personne souffrant d'une DTA, tout en pondérant ses souhaits à son opinion en temps réel, sa qualité de vie actuelle et celle de son soignant. Le déploiement bienfaisant des DA repose sur la capacité des acteurs impliqués à considérer cette démarche comme un processus de revalidation des ententes. Ce changement nécessaire de paradigme assure que l'actualisation de l'autodétermination des personnes atteintes d'une DTA ne se fasse pas au détriment de leur dignité, leur sécurité et leur intégrité. Une telle approche demeure à développer et à promouvoir pour les personnes atteintes d'une DTA au Québec. Des politiques de santé en ce sens et des repères normatifs spécifiques à cette clientèle doivent être rapidement mis de l'avant dans le contexte où le Québec associe le développement d'un régime de "directives médicales anticipées » à la Loi sur les soins de fin de vie.

\section{Mots clés}

directives anticipées, Alzheimer, fin de vie, politiques de santé, Québec, personnes âgées, incapacité décisionnelle, qualité de vie

\section{Abstract}

The Law on End of Life Care is now effective in Quebec. This law provides for a system of advanced medical directives. Respecting advanced directives (AD) issued in anticipation of incapacity can be a beneficial ethical way to ensure self-determination of the patient in a context where a person's incapacity is total and of short duration (e.g., terminal cancer). But what about when the loss of decisionmaking abilities is gradual and spread over a long period of time, as is the case of people with Alzheimer's type dementia (ATD)? Although the cognitive decline associated with $A T D$ legitimizes the use of $A D$ to promote selfdetermination of these people, relying solely on a person's wishes expressed verbally or in writing to their relatives or physician to guide care is an approach that can be ethically risky. A review of the literature on $A D$, a study of the clinical features associated with ATD, and a critical analysis of a fictitious clinical case will help demonstrate the need for developing a gradual approach to revalidate agreements that take into consideration the $A D$ of the person with a ATD while weighing their wishes and views in real time, their current quality of life and that of their caregiver. The beneficial deployment of $A D$ relies on the ability of the actors involved to consider this as a process of revalidation of agreements. This necessary paradigm shift will ensure that actualising the self-determination of people with ATD does not come at the expense of their dignity, safety and integrity. Such an approach remains to be developed and promoted for people with ATD in Quebec. Health policies that move in this direction, as well as specific normative benchmarks for this clientele, are needed rapidly in the current Quebec context, which is joining the development of a system of "advanced medical directives" with a Law on End of Life Care.

\section{Keywords}

advance directives, Alzheimer's, end of life, health policies, Quebec, seniors, decisional incapacity, quality of life 


\section{Exonération}

Les évaluations des examinateurs externes sont prises en considération de façon sérieuse par les éditeurs et les auteurs dans la préparation des manuscrits pour publication. Toutefois, être nommé comme examinateur n'indique pas nécessairement l'approbation du manuscrit par cet examinateur. Les éditeurs de BioéthiqueOnline assument la responsabilité entière pour l'acceptation finale et la publication d'un article.

\section{Disclaimer}

Reviewer evaluations are given serious consideration by the editors and authors in the preparation of manuscripts for publication. Nonetheless, being named as a reviewer does not necessarily denote approval of a manuscript by the reviewer; the editors of BioéthiqueOnline take full responsibility for final acceptance and publication of an article.

Affiliations des auteurs / Author Affiliations

${ }^{1}$ Équipe de psychogériatrie, CSSS Lucille-Teasdale, CIUSSS de l'Est-de-l'Île-de-Montréal, Montréal, Canada

\section{Correspondance / Correspondence}

Line Perreault, besper@videotron.ca

\section{Remerciements}

L'auteure souhaite remercier Dr Bryn Williams-Jones pour sa générosité, son soutien et son encadrement dans la réalisation de cette réflexion. Elle souhaite également remercier Dr Vanessa Chenel pour ses précieuses recommandations, le $\mathrm{Dr}$ Christophe Trivalle ainsi que le Professeur Alberto Bondolfi pour leurs commentaires constructifs sur le texte.

\section{Conflit d'intérêts}

Les études de maitrise de l'auteure ont été supervisées par Bryn Williams-Jones, Éditeur en chef de BioéthiqueOnline.

\section{Acknowledgements}

The author wishes to thank Dr. Bryn Williams-Jones for his generosity, his support and guidance in carrying out this reflection. She also wishes to thank Dr. Vanessa Chenel for her valuable recommendations, and Dr. Christophe Trivalle and Dr. Alberto Bondolfi for their constructive comments on this text.

\section{Conflicts of Interest}

The author's Masters was supervised by Bryn WilliamsJones, Editor-in-chief of BioéthiqueOnline.

\section{Introduction}

La démence de type Alzheimer (DTA) affecte progressivement les capacités cognitives et décisionnelles de l'individu qui en souffre. Ces personnes peuvent désirer émettre, au moment du diagnostic, des directives concernant les soins qu'elles souhaitent et ne souhaitent pas recevoir en cours d'évolution de la maladie et en fin de vie. Ces directives anticipées (DA) peuvent inclure des notions différentes et beaucoup plus larges que celles qui sont habituellement émises dans les cas où une incapacité totale, irréversible et de courte durée est anticipée, comme c'est le cas des victimes du cancer en fin de vie. Les notions de représentativité, de soins et de lieu de vie (exprimer dans quel contexte la personne souhaite recevoir ses soins, c'est-à-dire le domicile ou en institution) sont des thématiques sur lesquelles les personnes souffrant d'une DTA veulent se prononcer [1].

Au Québec, le mandat en cas d'inaptitude permet au majeur apte de prévoir sa représentativité en amont de ses incapacités décisionnelles [2]. La loi sur les soins de fin de vie (LSFV) vient maintenant permettre de fixer dans le temps le consentement ou le refus du majeur apte (en leur associant une valeur contraignante) pour certains soins fréquemment associés aux instants précédant la mort avec la création d'un régime de directives médicales anticipées (DMA) [3]. Mais que doit faire le décideur de substitution des DA émises par la personne atteinte de la DTA concernant les soins en cours de maladie et le lieu de vie? Dans le cas d'une personne qui souffre d'une DTA, le décideur de substitution peut se retrouver aux prises avec des DA émises il y a de cela plusieurs années, qui sont peu documentées, imprécises et souvent empreintes de fausses perceptions sur la maladie alors que la personne atteinte présente toujours certaines capacités décisionnelles. Que doit faire le décideur de substitution, lorsque le but des soins se heurte au respect des DA de l'être aimé? Doit-il donner préséance aux DA? 
La première partie de notre analyse aura pour objectif de sensibiliser le lecteur au concept éthique de DA en effectuant une revue de la littérature sur le sujet. L'origine, la définition, l'application, la portée et les limites de ces directives seront présentées et discutées de même que les multiples défis que leur déploiement génère en clinique chez les personnes atteintes d'une DTA. Nous regarderons par la suite les politiques de santé qui en font la promotion au Québec, tels que le mandat en cas d'inaptitude, la LSFV et le rapport Bergman [4]. Nous défendrons l'idée que d'honorer à la lettre les DA d'une personne souffrant d'une DTA pourrait lui porter préjudice et porter atteinte à sa dignité, son intégrité et sa sécurité. L'étude de cas de Mme Lapierre et de son neveu Jean sera utilisée pour illustrer ce fait et susciter une réflexion à l'égard des enjeux éthiques reliés à l'utilisation des DA chez les personnes atteintes d'une DTA dans le contexte du Québec. Nous déterminerons finalement : 1) quelle place le décideur de substitution devrait donner aux DA au moment où il doit décider pour l'autre, 2) les variables importantes à considérer au moment du déploiement des DA pour assurer à ces malades une vie et une fin de vie digne, et 3) les éléments clés pour l'élaboration future d'une politique de santé qui viendrait favoriser l'élaboration de DA réalistes chez les personnes atteintes d'une DTA.

Nous sommes d'avis que le décideur de substitution devrait effectivement tenir compte des directives anticipées formulées par la personne souffrant d'une DTA en début de maladie tout en modulant celles-ci aux volontés de la personne atteinte, sa qualité de vie et celle de son soignant pour assurer le caractère bienfaisant du respect de son autonomie qui, dans le cadre d'une DTA, s'affaisse graduellement (contrairement à d'autres types de pathologie). Une approche de revalidation des ententes accompagnée de repères normatifs demeure à définir et à vulgariser.

\section{Les directives anticipées}

La notion de DA repose sur le principe éthique du respect de l'autonomie et la doctrine légale du consentement éclairé [5]. Cette doctrine s'est développée au milieu du siècle dernier, moment où la médecine délaissait un modèle médical paternaliste pour évoluer vers un modèle de prise de décision autonomiste, c'est-à-dire centré sur la capacité décisionnelle du malade [6,7]. Les DA permettent à la norme du consentement éclairé de s'actualiser au moment où le malade perd ses capacités décisionnelles [8]. Cette visée est qualifiée de noble et honorable [9]. L'émergence des technologies qui prolongent la vie à la fin du siècle dernier a favorisé leur utilité en médecine [10]. La Convention d'Oviedo de 1997 pour la protection des droits de l'Homme et de la dignité de l'être humain à l'égard des applications de la biologie et de la médecine (article 2, 5 et 9) est l'un des premiers textes internationaux à souligner la pertinence de reconnaître les "souhaits précédemment exprimés " par un malade et à affirmer que les DA représentent une forme de consentement $[7,10]$. Afin de respecter la visée bienfaisante d'autodétermination que ce concept sous-tend, il faut s'assurer que la personne qui rédige ses directives ait eu accès à toute l'information nécessaire concernant la maladie et les soins associés, qu'il soit apte et stable émotionnellement au moment où il les émet. Les règles relatives au consentement libre et éclairé s'appliquent. Rappelons qu'un choix autonome est un choix intentionnel, fait avec compréhension et libre d'influence [5].

C'est en étudiant les définitions associées aux DA dans la littérature que nous remarquons la présence de différences conceptuelles au niveau des soins qu'elles englobent (fin de vie vs en cours de maladie) et de la temporalité (moment fixe d'émission et d'application vs la notion de processus). Hottois énonce que «Les directives anticipées sont des instructions données par anticipation concernant les traitements qu'on désire recevoir, ou (plus fréquemment) qu'on refuse, en fin de vie, au cas où l'on serait incapable d'exprimer ses volontés ou de prendre des décisions par soi-même » [11]. Sa définition met l'accent sur les directives émises à l'avance concernant les soins possibles en fin de vie (end of life strategies). Cette définition rejoint celle de Philips-Nootens qui utilise l'appellation " directives de fin de vie » [12]. Plusieurs autres auteurs, dont Singer, élargissent le concept à tous les soins médicaux qui s'imposent au malade au moment d'une incapacité décisionnelle et non seulement à ceux requis en fin de vie. "Advance care planning is a process whereby a patient, in 
consultation with health care providers, family members and important others, makes decisions about his or her future health care. This planning may involve the preparation of a written advance directive...the advance directive is invoked in the event that the patient becomes incapable " [8].

Singer évoque ici les conditions du mourir, mais également les conditions de soins en cours d'évolution de la maladie alors que le malade présente une incapacité décisionnelle [13]. « Advanced care planning ", " anticipated care planning engagement » et les appellations " planification préalable des soins " et "planification anticipée des soins" sont des termes équivalents, fréquemment retrouvés dans la littérature consultée. II est intéressant de noter que la Société canadienne d'Alzheimer fait référence aux « directives préalables » pour définir le concept. Ces termes englobent, contrairement à Hottois, les soins requis une fois que le malade n'a plus la capacité de s'exprimer en toute connaissance de cause et de porter un jugement éclairé à leur sujet et non seulement ceux requis en fin de vie. Nous portons également à l'attention la notion de processus soulignée par Singer qui permet d'introduire que ce concept n'est pas figé dans le temps et qu'il peut possiblement évoluer au fil des réalités vécues par le malade et des discussions entre les différents acteurs concernés (le malade, son décideur de substitution, ses proches et les soignants). Cette notion de processus est soulignée par d'autres auteurs, mais peu définie [14]. Elle est de notre avis une notion clé à retenir lorsqu'il est question d'élaborer et d'honorer les DA d'une personne souffrant d'une DTA. Nous y reviendrons plus tard en cours d'article. Pour les besoins de notre analyse, nous nous référerons à la définition de Singer lorsque nous évoquerons la notion de DA. Nous sommes d'avis que cette vision plus globale et plus dynamique correspond mieux aux besoins et à la réalité des personnes atteintes d'une DTA étant donné que la période d'inaptitude inhérente à cette maladie peut s'échelonner sur plusieurs années. II semble qu'au Québec, le régime de directives médicales anticipées, inclus dans la LSFV, s'inspire de la visée de Hottois [3].

Le concept éthique de DA s'actualise de plusieurs façons; soit en effectuant le choix d'un décideur de substitution verbalement ou par écrit (parfois aussi désigné dans la littérature par le terme représentant, mandataire, curateur, proxy et décisionnaire remplaçant), en rédigeant ses directives dans un document (qu'on désigne dans le langage populaire québécois et dans la littérature consultée de "testament biologique ») ou en partageant ses volontés lors d'une discussion avec son médecin ou un membre de sa famille (volontés exprimées) [5,15]. Ces façons de faire ne sont pas mutuellement exclusives ni inclusives, toutes les formes de combinaisons sont possibles. L'élaboration de ces directives implique également, possiblement plusieurs acteurs, soient : le malade (l'auteur), ses proches, ses soignants (professionnels et personnels), son décideur de substitution, les établissements de santé, le notaire et le législateur [8].

Le concept des DA s'illustre en clinique de multiples façons. La détermination de niveau d'intervention médicale (NIM) telle qu'on le fait dans plusieurs établissements du Québec en est un exemple ; il s'agit d'un formulaire utilisé dans les centres hospitaliers et les centres d'hébergement qui a pour but de clarifier avec le malade la visée des soins dans un contexte donné [16]. Le contrat d'Ulysse est notamment utilisé dans le domaine de la psychiatrie [17]; il permet à la personne souffrant d'une maladie mentale de laisser "son soi sain décider pour son soi malade " au moment où des choix de traitements sont offerts et que l'état psychologique du malade limite ses capacités décisionnelles [18]. En obstétrique, le plan de naissance permet à la femme d'énoncer en amont de l'accouchement les soins qu'elle souhaite recevoir durant l'accouchement, moment où les capacités décisionnelles sont influencées par la douleur et l'intensité de l'expérience ; comme le disent Philipsen et Haynes, "Birth plans and living wills are both advance directives that promote individualized care and improved outcomes for the individuals and their families »[19]. Ces différentes applications cliniques ont tous en commun la visée d'assurer une voix aux préférences exprimées par le malade en amont d'une condition médicale ayant le potentiel de rendre le consentement libre et éclairé impossible. Elles sont verbales ou écrites, ou les deux à la fois. Elles englobent la notion de représentativité, de soins en cours de maladie et en fin de vie. 
Les bénéfices rattachés à l'utilisation clinique des DA sont nombreux (améliore les soins, redonne du contrôle au patient sur sa maladie, actualise son autonomie décisionnelle, favorise le respect de son intégrité, centre les soins sur le malade, améliore la qualité de vie du malade et de son décideur de substitution, allège le fardeau des soignants et diminue le coût des soins) $[5,8,12,14,15,17,20]$. Effectivement, l'efficience des interventions des professionnels s'améliore lorsque les désirs des patients sont connus, le plan de soins est plus facilement défini (diminution des coûts en santé par épisode de soins) et plus proche des valeurs du patient.

Malgré ces nombreux avantages, on dénotait en 2012 au Canada que moins de $50 \%$ de la population avait déjà parlé à un de ses proches de ses DA [21]. Parmi ceux-ci : 73\% d'entre eux avaient fait le choix d'un représentant légal, $48 \%$ avaient spécifié les soins désirés et seulement $30 \%$ avaient informé leur médecin [21]. L'âge, le sexe, la composition de la famille (vivre en présence d'enfant diminue l'occurrence d'émettre des DA), le degré d'éducation, le revenu, la langue et la religion sont des facteurs qui influencent l'attitude des Canadiens face aux DA [14,20]. Les Canadiens âgés, caucasiens, éduqués, dont les revenus sont élevés sont ceux qui sont le plus sensibilisés à l'importance des DA. Le manque de popularité de ce concept chez nos concitoyens s'explique selon nous entre autres par le fait que le concept est notamment porteur d'ambiguïtés terminologiques, conceptuelles et méthodologiques comme nous l'avons mentionné auparavant. Notons également que le droit au Canada vient contribuer à cette confusion en évoquant de sept façons différentes la notion de DA [8]:

- « representation agreement » en Colombie-Britannique ;

- « personnal directive » en Alberta ;

- " health care directive » au Manitoba ;

- « power of attorney for personal care » en Ontario ;

- « consent agreement » en Nouvelle-Écosse ;

- " advance health care directive » à Terre-Neuve ; et

- « mandat en cas d'inaptitude » au Québec.

Le concept, les règles de rédaction et d'application varient aussi d'une province à l'autre. À cela, s'ajoute le fait qu'il est souvent reproché au DA de manquer de précisions et de flexibilité pour s'ajuster à la réalité en mouvance des patients [13,22]. Évoquons aussi que la rédaction des DA fait appel à nos croyances les plus profondes sur le sens de la vie [10]. Elles sont teintées des valeurs de l'individu qui les rédige et peuvent s'avérer contraires aux croyances de ceux qui devront les respecter (croyances culturelles et religieuses) [23]. Sans compter toutes les dérives possibles qui peuvent guetter le décideur de substitution au moment du déploiement des DA (difficultés d'interprétation et possibilité de conflit d'intérêts). Tous ces faits font certainement ombrage au déploiement du concept et font possiblement craindre le pire à ceux qui désirent en émettre [16,22]. À cela s'ajoute, comme le souligne Hirsch, le fait que la maladie en général cache un parcours " compliqué, incertain, hasardeux et contraint " [23] et qu'il est utopique de croire que la simple volonté de vouloir remplir un questionnaire ou tenir une discussion à ce sujet avec un proche est suffisante pour assurer l'autodétermination du malade. Formuler des DA demande du temps et une bonne dose de courage [15]. Le patient, ses proches et ses soignants n'ont pas toujours la force morale nécessaire pour aborder ce genre de discussion. Certains ne veulent tout simplement pas savoir et préfèrent s'éviter la souffrance associée à une telle réflexion. C'est aussi un choix à respecter [23]. La peur, la pitié et le mépris sont des réalités que l'on se doit de considérer et qui peuvent venir influencer le recours aux DA. II n'est pas évident que tous les patients ont la force morale nécessaire pour affronter la réalité de leur devenir. II en va de même pour les proches et les soignants. Anticiper l'avenir d'une maladie dégénérative tel que la maladie d'Alzheimer n'est certainement pas facile. 


\section{Les particularités cliniques de la démence de type d'Alzheimer}

La démence de type Alzheimer (DTA) est une maladie dégénérative qui touche approximativement 125000 individus au Québec, un chiffre qui doublera d'ici 2031 [24,25]. Cette démence évolue sur une longue période de temps (8 à 10 ans) [26]. Elle est caractérisée par 7 stades selon l'échelle de Reisberg (26). Les stades 1 à 3 sont les stades légers de la maladie, où les oublis et les autres déficits cognitifs s'installent graduellement. Les stades 4 et 5 sont les stades modérés, où les déficits cognitifs viennent clairement influencer le rendement occupationnel du malade au quotidien. Les stades 6 et 7 sont les stades avancés de la maladie ; ils sont marqués par des modifications de la personnalité, l'apparition des troubles moteurs, l'incontinence et le besoin de prise en charge constant du malade. Le médecin (généraliste ou spécialiste) est responsable de l'investigation, l'annonce du diagnostic et du suivi médical alors que d'autres professionnels de la santé (ex. : pharmacien, infirmier, travailleur social, ergothérapeute) peuvent être consultés par la suite pour guider la prise de décision concernant les soins (au niveau, entre autres, de la pharmacologie, de la qualité de vie et des capacités fonctionnelles) [25]. Chaque stade de la maladie est marqué par des défis cliniques différents. Les soins s'inscrivent dans une relation de "compagnonnage " [27]. Le défi consiste principalement à préserver une relation de dignité alors que la personne souffrant d'une DTA voit l'expression de sa personne s'effriter peu à peu. Les soins dans les stades légers (stade 1 à 3 ) et modérés (stade 4 et 5 ) visent la compensation des incapacités et le ralentissement de l'évolution de la maladie (ex.: par la pharmacologie) [28]. Les incapacités décisionnelles s'installent progressivement au fur et à mesure que la personne atteinte perd des habiletés cognitives, elles sont inévitables [20,22]. L'atteinte de la capacité à reconnaître la maladie (anosognosie) complexifie la tâche des soignants puisque la personne qui souffre d'une DTA ne perçoit pas sa perte d'autonomie, sa dépendance à l'autre et son besoin d'aide [29]. Une visée palliative [27] s'inscrit aux stades avancés de la maladie qui peuvent durer quelques années [5,17]. Cette condition est actuellement irréversible. Les complications normalement associées aux stades avancés de la maladie (dénutrition, infections) engendrent ultimement la mort de la personne atteinte [26].

La maladie d'Alzheimer touche directement l'autonomie des personnes qui en souffrent en affectant leur capacité à accomplir les tâches du quotidien [28], à prendre des décisions et à faire partie de la société active. "Cette triple fragilisation de l'autonomie (exécutionnelle, décisionnelle et participationnelle) de ces personnes explique leur singulière situation de vulnérabilité. Elle permet aussi de mieux comprendre la difficulté de la tâche de ceux qui les accompagnent au quotidien " [22]. Cette maladie affecte aussi toutes les personnes qui «constitue son entourage : son conjoint, les membres de sa fratrie, ses enfants, et petits-enfants et, plus largement, l'ensemble de ses proches » [30]. L'entourage doit voir au bien-être physique et moral de ces personnes. Leur rôle et leur besoin sont de plus en plus reconnus et documentés dans la littérature [30]. On sait maintenant que les questionnements moraux que les proches de la personne atteinte rencontrent (limites des devoirs filiaux, impact de la maladie sur l'identité de la personne, respect de la dignité, conciliation sécurité et liberté, décider pour l'autre et comment maintenir les personnes atteintes dans la communauté) diffèrent de ceux des professionnels de la santé [30]. II devient essentiel pour la société de les soutenir adéquatement, car ces questionnements contribuent au fardeau de ces aidants alors que leur complicité est si précieuse pour assurer le bien-être des personnes souffrant d'une DTA $[28,30]$.

Kerrigan et Ormerod soulignent la légitimité pour les personnes atteintes d'une DTA de vouloir souscrire à l'émission de DA étant donné l'implication prévisible et progressive d'incapacités décisionnelles [20]. Les personnes souffrant d'une DTA peuvent désirer émettre, au moment du diagnostic, des directives concernant les soins qu'elles souhaitent et ne souhaitent pas recevoir en cours d'évolution de la maladie et en fin de vie. Ces directives anticipées (DA) peuvent inclure des notions différentes et beaucoup plus larges que celles qui sont habituellement émises dans les cas où une incapacité totale, irréversible et de courte durée est anticipée comme c'est le cas des personnes qui souffrent du cancer en phase terminale. Les notions de représentativité, de soins, de participation 
à la recherche et de lieu de vie (exprimer dans quel contexte la personne souhaite recevoir ses soins, c'est-à-dire le domicile ou en institution) sont des thématiques sur lesquelles les personnes qui souffrent d'une DTA veulent et devraient se prononcer $[1,22,30]$. Encore faut-il que la personne atteinte ait démontré suffisamment de compétences pour comprendre la complexité des enjeux quant aux volontés qu'elle s'apprête à énoncer. Le tout devient possible si le diagnostic est posé au début de la maladie. C'est l'une des raisons pour lesquelles les recherches actuelles dans le domaine de l'Alzheimer visent, entre autres, à mieux comprendre et identifier les stades précoces de la maladie [31]. II faut aussi que la possibilité de réfléchir à ces enjeux soit convenablement offerte à la personne atteinte en début de maladie, que les professionnels de la santé et les organismes sans but lucratif qui accompagnent ces personnes y soient sensibilisés et prêts à les accompagner et que la société, le législateur, les familles et le corps médical soient en mesure de les entendre et de prôner une gestion bienfaisante de ses souhaits. Dans les cas où le diagnostic est émis plus tardivement, LopezTourres considère que la personne atteinte d'une DTA ne doit pas être écartée de la réflexion sur ses soins [28]. Nous rajouterons à cela qu'elle doit être accompagnée et nous élaborerons plus tard les balises essentielles à un accompagnement bienfaisant.

\section{Politiques de santé du Québec associées aux directives anticipées pour une personne atteinte d'une démence de type d'Alzheimer}

Nos recherches nous ont permis de constater que les lois actuellement en vigueur au Québec prévoient la possibilité d'énoncer à l'avance des substituts au majeur en prévision de son inaptitude [2,32]. Le mandat en cas d'inaptitude est un moyen que le législateur a institué à l'occasion de la réforme du Code civil du Québec en 1990 pour assurer le respect des volontés d'un majeur, en lui permettant de prévoir sa représentativité en cas d'inaptitude. "L'esprit qui sous-tend la législation est de respecter, dans toute la mesure du possible, la volonté qu'aurait pu exprimer, même tacitement, la personne concernée avant la survenance de son inaptitude » [2]. La mention " en tenant compte, dans la mesure du possible, des volontés que cette dernière a pu manifester » contenue dans l'article 12 du Code civil du Québec, nous apparaît tout de même vague considérant que plusieurs interprétations sont possibles. "A pu manifester » quand [33]? Au moment même où la décision doit se prendre, au moment de l'émission de ses souhaits, ou les deux ? Ces représentants, dans l'esprit du même article, ont droit à toute l'information nécessaire (fait exception aux règles habituelles à la confidentialité) pour donner un consentement libre et éclairé. Les tribunaux ont préséance sur le représentant légal s'il est impossible d'agir, s'il manifeste un refus injustifié et si le majeur inapte lui-même manifeste un refus catégorique [32]. Ce mandat (notarié ou un écrit fait devant 2 témoins) donne au mandataire désigné le pouvoir d'administrer les biens ou de prendre soin de la personne inapte, ou les deux à la fois [12]. En l'absence de représentant légal, l'article 15 du Code civil du Québec prévoit que le conjoint peut agir comme décideur de substitution, qu'il soit marié, en union civile ou en union de fait, ou, à défaut de conjoint ou en cas d'empêchement de celui$\mathrm{ci}$, un proche parent ou par une personne qui démontre pour le majeur un intérêt particulier au moment du consentement à des soins, peut décider pour lui [33].

La LSFV viendra bousculer bien des mœurs au Québec dans les prochains mois. Cette loi, sanctionnée le 10 juin 2014 par l'Assemblée nationale du Québec [3], est en vigueur depuis décembre 2015. Elle réglemente les droits, l'organisation et l'encadrement des soins palliatifs offerts aux personnes en fin de vie, ainsi que l'aide médicale à mourir. Cette loi a été élaborée pour permettre aux personnes aptes désirant mettre fin à leur souffrance secondaire à une maladie incurable telle le cancer de le faire. Elle nous apparaît peu spécifique à la réalité des personnes atteintes d'une DTA. Ce fait mérite d'être souligné, car cette loi ne permet pas aux personnes atteintes d'une DTA d'y avoir recours (article 51, LSFV). En effet, il est prévu qu'une telle demande doit être faite par un majeur apte, en fin de maladie alors que ses souffrances (physiques et morales) sont incurables, ce qui laisse la personne atteinte d'une DTA qui meurt de cette maladie incapable d'avoir recours à ce droit étant donné son inaptitude au moment des derniers stades de la maladie. 
Peut-on parler d'injustice pour ce sous-groupe de personnes ou de protection des grands vulnérables contre toute forme d'homicide et de génocide [28,34]? Que ferons-nous comme société des souhaits de fin de vie de ces personnes ? La LSFV au Québec inclut également la mise en place d'un régime " des directives médicales anticipées » [3]. Le régime prévoit la rédaction de volontés qui respectent la visée d'un consentement libre et éclairé, faite devant notaire ou devant témoins avec des modalités de révision et l'assurance qu'elles sont en tout temps révocables. À la lecture des informations disponibles sur le site internet du Gouvernement du Québec (articles : 51-64) [3], nous constatons que le concept de "directives médicales anticipées » est circonscrit et que seules les volontés qui respecteront la forme prescrite par la loi pourront être inscrites au registre. L'appellation " médicales » introduite entre les mots "directives » et "anticipées " annonce une forme restrictive du concept éthique de directives anticipées. Les «directives médicales anticipées " n'englobent que les directives rattachées à des soins techniques et pharmacologiques spécifiques (ex. : la réanimation cardiaque, le recours à un respirateur, la dialyse et à une alimentation et une hydratation artificielles) précédant la mort. Ce régime prévoit la rédaction écrite de volontés spécifiques. Le déploiement des DMA (intégration au plan de soin) sera sous la responsabilité des professionnels de la santé. II est clairement précisé que le régime des DMA ne pourra pas servir à formuler une demande d'euthanasie. Plusieurs des notions pertinentes aux DA des personnes atteintes d'une DTA n'y sont pas incluses telles que le choix d'un lieu de vie. Il est de plus clairement précisé sur le site du Gouvernement du Québec que les volontés relatives aux soins exprimées dans un mandat en prévision d'une inaptitude ne seront pas considérées comme des DMA au sens de la loi (article 62 de la LSFV) [3]. Cette loi donne une valeur contraignante aux volontés exprimées dans le régime, ce qui s'avère néanmoins une première au Québec.

La pertinence de prévoir la représentativité du majeur inapte fait consensus pour la France, la Suisse, la Belgique, les Pays-Bas et les États-Unis [12,13]. Bien qu'il n'est pas prévu dans le présent article de faire une revue exhaustive et comparative de l'application pratique du concept éthique des DA à travers le monde, nous soulignons le fait que tous ces pays reconnaissent également la possibilité d'émettre des DA concernant les soins précédant la mort. Seulement la Belgique, la Suisse et les Pays-Bas associent actuellement une valeur contraignante à ces souhaits [35-37]. Les DA représentent ainsi pour ces pays une finalité. Les États-Unis et la France perçoivent et utilisent plus précisément les DA comme un moyen clinique de favoriser la discussion [7,13,35,38]. Notons qu'il est possible en Belgique et aux Pays-Bas d'anticiper une euthanasie [35]; voir le cas de l'écrivain Hugo Claus qui a demandé l'euthanasie alors qu'il souffrait d'une DTA [39].

L'émergence de la reconnaissance des DMA pourrait avoir des implications pour les personnes atteintes d'une DTA au Québec étant donné que cette notion nécessitera d'être expliquée au grand public et que la visée bienfaisante de ce concept sera popularisée, mais elles demeurent difficiles à prévoir. Nous estimons problématique que le dossier des DA se développe au Québec parallèlement et inclusivement avec la loi concernant les soins de fin de vie. La population viendra certainement associer à la notion de DMA les besoins rattachés spécifiquement aux personnes qui désirent mettre un terme aux soins précédant la mort délaissant ainsi tous les aspects sociaux du soin si précieux aux personnes vivant une longue période d'incapacité décisionnelle comme c'est le cas des personnes souffrant d'une DTA. Nous déplorons que la notion de lieu de soin soit par exemple complètement oubliée dans ce contexte. Cette vision s'avère loin des besoins des personnes qui souffrent d'une DTA et qui désirent émettre en prévision de leur inaptitude leurs souhaits face aux multiples soins qui s'offriront à eux en cours de maladie (ex. : protocole de recherche, lieu de vie, interventions médicales mineures). De plus, cette loi vient fixer le concept des DA dans le temps (en conformité avec la vision de Hottois), ce qui ne convient pas selon nous aux besoins des personnes atteintes d'une DTA, comme nous l'expliquerons dans la section suivante.

Le rapport du comité d'experts en vue de l'élaboration d'un plan d'action national pour la maladie d'Alzheimer, communément appelé le « rapport Bergman », soulignait déjà en 2009 l'importance de développer une stratégie de sensibilisation grand public visant l'évolution naturelle de la maladie, et 
l'élaboration de " directives de fin de vie anticipées » [4]. Ce rapport reconnaissait l'importance d'offrir aux familles de la formation et du matériel éducatif concernant la fin de vie des personnes atteintes de la maladie d'Alzheimer. II soulignait la pertinence de former les professionnels et de voir à la promotion de ce type de démarche dans tous les établissements du réseau de la santé québécois. Mes collègues et moi avions beaucoup d'attentes concernant le déploiement des recommandations de ce rapport, malheureusement 6 ans plus tard, la Société québécoise d'Alzheimer confirme lors d'une entrevue téléphonique réalisée en novembre 2014 que cette recommandation se fait toujours attendre. Cela est déplorable étant donné que la DTA est décrite par plusieurs comme une épidémie silencieuse et une menace à la santé publique de nos populations $[28,40]$. Soulignons qu'en 2001 , les Français sont les premiers à avoir lancé une offensive nationale pour contrer les effets sociétaux de cette maladie. Les Pays-Bas ont suivi en 2008, le Royaume-Uni en 2009, le Danemark et les ÉtatsUnis en 2010 [40]. Le président de la rencontre du G8 au Sommet sur la démence de 2013, David Cameron, affirmait la volonté des partenaires du G8 de vouloir formuler un plan global à ce niveau [40]. Nous constatons que le Québec prend un retard considérable dans ce domaine et qu'il serait temps d'agir. Précisons qu'en France, il est documenté que les personnes qui souffrent d'une DTA utilisent peu les DA même si elles sont reconnues légalement depuis avril 2005 grâce à la loi Léonetti [7]. Cela vient renforcer selon nous le constat que le concept mérite d'être mieux défini et mieux accompagné.

Des lignes directrices claires et spécifiques à la réalité de ces personnes demeurent à développer pour assurer le déploiement bienfaisant des DA chez ce groupe d'individus. Nous sommes d'avis, tout comme Singer, qu'il faudrait voir au développement de repères normatifs qui énonceraient les meilleures pratiques en ce sens [8]. II faudrait également encourager le développement d'incitatifs. Cela pose indirectement la délicate question de la rémunération du professionnel qui aborde ces questions avec le patient. On voit dans la littérature en provenance des États-Unis que la question est soulevée et débattue $[6,15]$. La rémunération du professionnel de la santé devient plus évidente dans un contexte où les DA sont reconnues par la loi, ce qui est le cas dans certains états américains. Une bonne façon d'encourager l'implication des médecins du Québec au niveau de la rédaction des DA serait de revoir leur rémunération en ce sens étant donné que la littérature nous indique que des DA élaborées conjointement avec un professionnel de la santé sont plus spécifiques [8]. Mais est-ce vraiment nécessaire dans un contexte d'optimisation des ressources et de rationalisation des coûts de santé au Québec? Cette responsabilité ne pourrait-elle pas être confiée à d'autres professionnels (travailleurs sociaux, infirmières, etc.) ou simplement à des organismes communautaires ou à un organisme à but non lucratif comme les sociétés d'Alzheimer? La diversification des moyens pour sensibiliser la personne atteinte et ses proches à la pertinence d'élaborer des DA nous apparaît de toute façon évidente dans un contexte où l'État se désinvestit des soins donnés aux personnes âgées [41].

Une recherche sur le site web de la Société d'Alzheimer nous apprend que le 16 avril est la Journée nationale de la planification préalable des soins au Canada. Ce site web encourage ainsi les personnes atteintes d'une DTA à s'y référer. Les outils disponibles sur www.advancecareplanning.ca nous apparaissent peu spécifiques à la réalité de ces personnes. Nous sommes d'avis que des guides de pratique et des outils cliniques demeurent à construire et pourraient faire l'objet de subvention de recherche en ce sens par cet organisme. Les organismes à but non lucratif sont de notre avis des acteurs de choix pour assurer le développement de ces balises, car ils connaissent bien la maladie et les défis qu'elle fait vivre aux personnes atteintes et à leur famille. Ces organismes ont un pouvoir politique différent étant donné qu'ils sont axés sur la qualité de vie de ces personnes et qu'ils n'ont aucun intérêt financier, comme cela pourrait être le cas des médecins. Leur pouvoir d'influence auprès du gouvernement n'est certainement pas à négliger. 


\section{L'histoire de cas de Mme Lapierre}

Nous utiliserons maintenant une histoire de cas pour enrichir notre réflexion. Celle-ci est inspirée directement de notre pratique clinique (de plus de 25 ans) en tant que professionnelle de la santé dans le domaine de la gériatrie. Elle demeure fictive tout en rassemblant tous les éléments pertinents des cas réels que nous avons eu la chance de rencontrer. Elle permettra d'illustrer à quel point le respect strict des DA d'une personne âgée souffrant de la maladie d'Alzheimer peut poser problème aux proches, aux équipes de soins, à la société et à la personne atteinte.

Madame Lapierre est âgée de 78 ans. Elle souffre d'une DTA depuis plus de 5 ans. Elle est en investigation pour une arythmie et il est possible qu'un stimulateur cardiaque lui soit installé dans les prochains jours. Elle est également en attente d'une chirurgie pour une cataracte à l'œil gauche. Elle vit seule dans un appartement depuis la mort de son conjoint. Elle n'a pas eu d'enfant. Elle aurait fait promettre à son neveu Jean au moment du diagnostic qu'il s'occuperait toujours d'elle et que jamais il ne la " placerait » dans un centre d'hébergement pour personnes âgées du réseau public québécois. Ses souhaits par rapport à son devenir ne sont pas documentés par écrit et seul le neveu peut en témoigner.

Depuis quelques mois, la perte d'autonomie de Mme Lapierre s'accentue et met sa capacité à prendre soin d'elle-même en péril. Elle est jugée non sécuritaire par son médecin de famille et son équipe de soins (infirmière, travailleuse sociale et auxiliaires) du Centre local de services communautaires (CLSC). Son neveu Jean a récemment procédé au débranchement de sa cuisinière de sorte que sa tante dépend des visites quotidiennes d'une voisine pour manger un repas chaud. Les auxiliaires du CLSC visitent Mme Lapierre tous les matins pour superviser la prise de la médication. Des voisins ont vu Mme Lapierre se promener la nuit dans les corridors de l'immeuble. Elle aurait accidentellement uriné dans le hall d'entrée de l'immeuble il y a deux semaines. Le neurologue traitant reconnaît l'inaptitude de madame Lapierre et détermine que sa perte d'autonomie est compatible avec le début du stade avancé de la maladie (stade 6 sur une possibilité de 7). II recommande un hébergement dans une ressource correspondant à ses besoins.

Son neveu, ayant promis de ne jamais « la placer », ne peut l'amener vivre chez lui et il ne veut pas aller vivre avec elle. II refuse de briser sa promesse et demande aux intervenants du CLSC d'ajouter de l'aide à domicile supplémentaire, ce qui n'est pas possible étant donné que Mme Lapierre refuse l'aide qu'on lui propose se croyant autonome pour tout (relié à l'anosognosie).

Les intervenants du CLSC constatent qu'ils ont déployé tous les moyens disponibles pour repousser les frontières de l'hébergement sachant qu'elle désirait rester chez elle. Ils ont respecté autant que possible son "droit au risque » [30]. Ils souhaitent maintenir le lien de confiance avec l'usagère et le neveu Jean, qui est à la fois le décideur de substitution, le seul proche parent et le seul aidant de cette dame. Ils ne savent plus comment faire. Ils sont déchirés à l'idée d'interpeller le tribunal pour imposer l'hébergement. Ils souhaiteraient revoir avec le neveu sa promesse pour préserver la dignité et l'intégrité de cette dame. Le neveu refuse, car il ne veut pas trahir sa tante. Les intervenants et le neveu sont tiraillés entre le désir de vouloir respecter les volontés de cette dame (principe d'autonomie) et le désir de vouloir préserver sa sécurité, sa dignité, et son intégrité (principe de bienfaisance/non malfaisance). 
Les intervenants du CLSC se demandent ce que comprenait vraiment Mme Lapierre et son neveu de la DTA au moment de la formulation de ses souhaits et de l'engagement moral du neveu. Le neveu avait-il eu la chance d'anticiper tout ce que sa promesse engageait ? Mme Lapierre aurait dû être en mesure de comprendre au moment de l'émission de ses DA qu'elle ne pourrait demeurer seule à domicile jusqu'à la fin de sa maladie. La situation clinique aurait certainement évolué différemment si Mme Lapierre et son neveu avaient compris, au moment de la formulation des DA, que celle-ci serait réévaluée en cours de maladie en fonction de l'évolution de la maladie, des évènements et de la détérioration de ses capacités décisionnelles tout en considérant ses directives anticipées, son opinion actuelle de même que sa qualité de vie et celle de son soignant.

Cette histoire de cas nous sert maintenant d'ancrage pour discuter :

- de la pertinence d'élargir la notion de DA aux soins requis en cours d'évolution de la maladie compte tenu du long caractère évolutif (plusieurs années) des incapacités décisionnelles associées à la DTA;

- des limites de l'utilisation des DA auprès des personnes souffrant d'une DTA dans le contexte actuel au Québec;

- de la nécessité d'instaurer un accompagnement professionnel adéquat au moment de la rédaction et de l'application des DA;

- du besoin de mettre en place un processus de revalidation des ententes compte tenu du long caractère d'évolution de la maladie [28]; et

- du bien-fondé de promouvoir le développement de politiques de santé et de repères normatifs pour permettre un déploiement bienfaisant de ce concept pour les personnes souffrant d'une DTA.

\section{Vers une utilisation bienfaisante des directives anticipées chez les personnes atteintes d'une démence de type Alzheimer}

Mme Lapierre a émis le souhait à son neveu Jean qu'il s'occupe d'elle et qu'il lui permette de continuer sa vie à la maison. Ces volontés exprimées verbalement à son neveu représentent une forme possible de DA tel que nous l'avons décrit précédemment dans le texte [5]. Le neveu Jean s'avère son décideur de substitution. Mme Lapierre a donné certains repères à ses proches en terme de représentativité et de lieu de vie. Mais elle est restée sans indication concernant ses volontés face à de possibles interventions médicales (DA incomplètes). Elle doit subir une chirurgie pour une cataracte et elle aura possiblement besoin de se faire installer un stimulateur cardiaque. Ces interventions auront des implications sur sa qualité de vie à court et moyen terme. Selon le cadre législatif en vigueur au Québec, c'est son neveu Jean qui devra se prononcer pour elle et prendre la décision qui s'impose dans le meilleur intérêt de sa tante. L'article 12 du Code civil du Québec [33] mentionne:

Celui qui consent à des soins pour autrui ou qui les refuse est tenu d'agir dans le seul intérêt de cette personne en tenant compte, dans la mesure du possible, des volontés que cette dernière a pu manifester. S'il exprime un consentement, il doit s'assurer que les soins seront bénéfiques, malgré la gravité et la permanence de certains de leurs effets, qu'ils sont opportuns dans les circonstances et que les risques présentés ne sont pas hors de proportion avec le bienfait qu'on en espère.

Le modèle du meilleur intérêt est critiqué par Gzil qui affirme que les décisions du décideur de substitution chez les personnes souffrant d'une DTA devraient plutôt être basées sur le modèle du jugement substitué, c'est-à-dire ce que le malade "aurait décidé s'il avait été en position de 
choisir » [22,30]. Cette vision est partagée par Philips-Nootens [12]. High défend quant à lui que ce qui est essentiel pour la personne atteinte d'une DTA, ce soit que ses proches soient consultés " parce que les décisions expriment les valeurs partagées au sein de la famille » et que le modèle de prises de décision retenu (jugement substitué vs meilleur intérêt) importe peu [22,30]. Pour cet auteur, il s'avère implicite que les familles intègrent au moment des décisions les préférences connues de la personne atteinte, son meilleur intérêt en temps réel, mais aussi les préférences et les intérêts des tiers qui sont impliqués.

L'histoire de cas de Mme Lapierre nous fait aussi prendre conscience de l'importance que revêt le lieu de vie pour les personnes atteintes d'une DTA [30]. Mme Lapierre avait émis le souhait de poursuivre sa vie à la maison. Dans le contexte d'une DTA, le lieu de vie des personnes atteintes est aussi le lieu de soin. Mais est-il vraiment raisonnable d'inclure la notion de lieu de vie aux DA? Soulignons pour débuter que le droit considère que le milieu où les soins sont donnés représente en soi, un soin pour le majeur inapte. Deleury et Goubau dans leur traité sur l'inviolabilité rappellent la notion englobante et complexe que sous-tend le terme de soin pour le droit [32]. Ils mentionnent qu'il doit être entendu dans son sens "générique tant du point de vue de son objet que de la nature des actes qu'il recouvre ». Pour eux, les soins comprennent tous les soins de base, telles l'alimentation et l'hydratation, mais aussi l'usage des contentions (physiques et pharmacologiques), les mesures d'isolement d'un patient et le choix d'un lieu de vie. "L'hébergement peut être qualifié de soin au sens de l'article 11 Code civil du Québec » [32]. Cette vision est également partagée par d'autres juristes qui considèrent que l'ordonnance d'hébergement fait partie de l'ordonnance de soins [18]. C'est ainsi qu'une ordonnance de traitement peut être suivie d'une ordonnance d'hébergement [18]. Le droit considère l'hébergement comme un soin qu'on peut prescrire, obliger et même forcer. II devient légitime dans ce contexte que les personnes qui souffrent d'une DTA comme Mme Lapierre souhaitent apporter des précisions sur la notion de lieu de vie lorsqu'elles émettent des DA. Nous rajouterons à cela le fait qu'en $2011,75 \%$ des décès au Canada se produisaient à l'hôpital et dans les établissements de soins de longue durée, et ce, malgré que plus de $60 \%$ des Canadiens préféreraient décéder à domicile [42]. Un sondage CROP réalisé en 2010 pour l'AQESSS (Association québécoise des établissements de santé et services sociaux) indiquait que $72 \%$ des Québécois âgés entre 50-64 ans souhaitaient vivre à domicile (avec des soins publics ou privés) dans l'éventualité où ils ne pourraient subvenir à leurs besoins [43].

Nous estimons donc, dans ce contexte essentiel, que le décideur de substitution ait l'occasion de discuter en amont des incapacités décisionnelles de la personne qu'il représente de ses volontés en matière de lieu de vie considérant que les stades avancés d'une DTA nécessitent une prise en charge constante de la personne et cela bien avant qu'elle ne soit en situation de fin de vie. Sans compter que les proches ne sont pas toujours en mesure d'offrir et de prendre en charge les soins. Mme Lapierre aurait pu manifester son désir à son neveu de vivre chez elle le plus longtemps possible tout en déterminant avec lui l'endroit de son choix au moment où son état nécessiterait une prise en charge constante ou du moins lui énoncer les enjeux (valeurs et prérequis) importants à considérer. Nous pourrions ainsi prévenir des promesses irréalistes, comme celles faites par le neveu de Mme Lapierre, et alléger le fardeau moral des aidants. C'est pour ces raisons que nous sommes d'avis que le lieu de vie doit faire partie intégrante des DA des personnes atteintes d'une DTA. Bien évidemment, des repères normatifs ou un accompagnement seront nécessaires pour assurer l'émission de directives réalistes. Rappelons que ce sont des enjeux reliés à la sécurité et à l'intégrité qui stimulent les professionnels de la santé et les décideurs de substitution à faire basculer les soins du domicile vers une ressource d'hébergement. Ce fait mérite selon nous d'être expliqué à la personne atteinte d'une DTA et à son décideur de substitution en début de maladie.

Philips-Nootens indique qu'un soin forcé constitue une atteinte au droit à l'inviolabilité de la personne et qu'il sera imposé au majeur inapte par le droit que pour des raisons de protection [18]. Le principe de bienfaisance prédomine alors et l'aide du tribunal est requise [12]. Mais Mme Lapierre pourrait s'y opposer! Bien que « le droit reconnaisse au majeur apte le droit absolu de refuser des soins quelles 
qu'en soient les conséquences sur sa qualité de vie et sur sa vie elle-même »[32], Deleury et Goubau dénoncent que le droit au refus soit malheureusement rendu presque impossible pour le majeur inapte et souhaitent qu'il en soit autrement. Trop souvent, selon eux, les tribunaux jugent le bien-fondé du refus en passant par le prisme d'un test portant sur l'aptitude. II y aurait pour ces juristes, deux aptitudes à documenter : l'aptitude à consentir à des soins et l'aptitude à les refuser. Le souhait de Mme Lapierre de demeurer chez elle peut s'actualiser tant que sa sécurité n'est pas menacée. Un ordre de Cour, qu'elle ne pourra pas contester (par manque de capacité), viendra mettre fin à sa volonté à moins que son neveu et son équipe de soins ne puissent pas faire cheminer Mme Lapierre vers un hébergement volontaire. Soulignons tout de même que le majeur inapte jouit d'un droit de refus absolu pour ce qui est des soins non requis par son état de santé [32]. Un acte non consensuel demeure un acte criminel, c'est aux soignants de déployer tous les moyens possibles pour aider la personne atteinte à comprendre en temps réel la pertinence des soins qu'elle doit recevoir et les rendre non agressants [20]. L'intervention du tribunal est également requise si le représentant légal, le mandataire ou un proche d'une personne inapte devait refuser un traitement jugé opportun à celle-ci [12]. Refuser l'hébergement d'une personne inapte qui a besoin de soin pour assurer sa dignité et sa sécurité équivaut à un refus de traitement injustifié. Le consentement du décideur de substitution à l'hébergement d'une personne inapte " ne vaut qu'en l'absence d'opposition de cette personne et son consentement aux soins est écarté si le patient oppose à ceuxci un refus catégorique »[12].

L'utilisation des DA dans le cas d'une DTA nous fait réaliser le fait que certaines directives anticipées peuvent devenir en cours de maladie désuètes et dangereuses, comme c'est le cas de la volonté de madame Lapierre de vouloir demeurer à la maison. Ce phénomène est particulier à ce sous-groupe de personnes et s'explique par le fait que cette maladie fait vivre une longue période d'incapacité décisionnelle dont les degrés fluctuent en fonction de la gravité de la maladie. Le décideur de substitution porte toujours le doute de la pertinence et du moment d'application de chaque directive [8]. En début de maladie, la personne souffrant d'une DTA a besoin d'être accompagnée dans ses décisions alors qu'aux stades intermédiaires et avancés, cet accompagnement s'intensifie et dépend du type de décision à prendre [30]. Les difficultés éprouvées par les décideurs de substitution lorsqu'ils doivent prendre des décisions pour la personne souffrant d'une DTA sont bien documentées dans la littérature [7,30]. Beauchamp et Childress indiquent que le décideur de substitution doit alors tenter de respecter les DA tout en apportant, si nécessaire, un jugement de substitution qui va dans le sens du meilleur intérêt de la personne qu'il représente [5]. Hirsch reconnaît le double fardeau du décideur de substitution qui est, dans bien des cas, à la fois décideur et à la fois soigneur [23]. II indique qu'il doit être en mesure de se substituer à la personne souffrant d'une DTA au moment de prendre une décision pour elle [27]. Ces propos vont dans le sens de Gzil [30]. Le recours aux tribunaux est parfois nécessaire pour assurer le meilleur intérêt de ces personnes, lorsque les soins nécessaires ne peuvent être possibles que par imposition.

La responsabilité des soins offerts aux personnes d'une DTA revient à leurs soignants (le terme soignant inclut ici les proches, le décideur de substitution et les professionnels de la santé). "La perte d'autonomie psychique doit conditionner les modalités pratiques d'une préservation de la personne, envers et contre tout, dans ses convictions, ses exigences, ses aspirations et ses droits ॥ [27]. Hirsch évoque ici la responsabilité fiduciaire des soignants. Cette responsabilité annonce une obligation dans la relation de soin, qui vise le bien-être et la protection des intérêts de la personne atteinte. Elle est basée sur la confiance qui se renouvelle lors de chaque geste de soin témoignant d'une relation respectueuse et loyale. C'est à ses soignants que revient la responsabilité de la rencontre avec la personne malade. Le soignant se voit ainsi imputer la responsabilité de la réponse aux besoins de l'autre, mais aussi de la poursuite de l'actualisation de son être (actualisation de ses convictions, ses aspirations et ses droits). Gzil reconnaît la double obligation morale des professionnels et des familles envers la personne atteinte, soit celle de le protéger et celle de respecter son autonomie et ses libertés, il nous éveille à toute la complexité de ce rôle [22,30]. Plusieurs soignants pourraient ne pas avoir les compétences nécessaires à cette tâche. II peut être sensé et louable de vouloir relayer cette 
responsabilité à un tiers institutionnel. Cela n'implique pas que le soignant se désengage de cette responsabilité sans l'exclure complètement. Si l'intégrité, la sécurité et la dignité de la personne atteinte sont menacées par l'actualisation de ses DA, c'est à ses soignants au sens large que revient le fardeau de s'adresser à la Cour pour arrêter ou imposer un soin. Ne pas honorer cette responsabilité serait de notre avis une faute éthique majeure qui s'apparenterait à l'abandon. N'avons-nous pas comme société la responsabilité morale de ne pas abandonner les plus vulnérables? Voilà entre autres pourquoi il ne serait pas éthique de laisser Mme Lapierre continuer à vivre seule dans son logement.

II est souhaitable que le décideur de substitution choisi soit libre de conflits d'intérêts à l'égard de la personne qu'il représente, qu'il ait été informé de son choix et qu'il reconnaisse que les décisions qu'il prendra au nom de la personne qu'il représentera seront basées en fonction du meilleur intérêt de la personne atteinte et non en fonction de son intérêt propre [12]. II est évident que ce représentant aura besoin du soutien de professionnels en cours de mandat, car il existe tout de même le risque que le représentant base les décisions qu'il prendra pour la personne atteinte sur ses propres valeurs et désirs en partie ou en totalité. Ou tout simplement en se basant uniquement sur des repères expérientiels du passé du malade pour justifier une décision qui ne tiendrait pas compte de la qualité de vie présente de la personne comme c'est le cas du neveu de Mme Lapierre. Exprimer ses directives anticipées verbalement au décideur de substitution en amont de son inaptitude, sans trace écrite et sans témoin autre que le principal intéressé, comme c'est le cas pour Mme Lapierre, comporte plusieurs risques. En effet, la personne qui entend ses volontés pourrait mourir, perdre la mémoire, modifier ses dires ou simplement les ignorer [6].

L'élaboration de DA, aussi louable soit-elle, pour assurer la promotion de l'autodétermination du malade sous-tend, dans une visée bienfaisante, que le malade soit capable de comprendre parfaitement comment la maladie qui l'affecte le changera. Dans le cas de la maladie neurologique dégénérative évoluant sur plusieurs années, comme c'est le cas dans la DTA, il devient impossible de savoir avec certitude comment la maladie changera l'humain qui en sera touché [17]. II devient hasardeux et même impossible de tout prévoir [7]. Mme Lapierre n'avait certainement pas réalisé, au moment d'émettre ses directives à son neveu, qu'elle ferait de l'errance la nuit et qu'elle aurait des incontinences dans le hall d'entrée de son immeuble lorsqu'elle a fait promettre à son neveu qu'elle souhaitait finir ses jours à la maison. Bien sûr, ces personnes suivent une certaine évolution qui est commune à toutes les victimes de cette condition, mais une grande partie de l'actualisation de la maladie demeure difficilement prévisible et elle varie d'une personne à l'autre (exemple : ils ne sont pas tous désinhibés, certains sont apathiques, d'autres plus énergiques) [7,30]. À cela s'ajoute le fait que la DTA évolue sur plusieurs années [22,30], la personne atteinte peut être victime d'autres problèmes de santé, sa situation sociale est susceptible de subir des transformations (exemple : mort d'un être cher, manque d'argent), la science pourrait changer entre le moment d'émettre des DA et leur actualisation en temps réel, permettant d'autres avenues thérapeutiques non préalablement anticipées [7].

Le facteur temps et l'incapacité de prédire avec certitude l'avenir viennent influencer l'élaboration et l'application des DA auprès des personnes atteintes d'une DTA [10]. Non seulement l'avenir est difficilement prévisible, mais il est en général associé à un devenir peu reluisant. Sokolowski évoque que cette maladie est communément décrite comme une terrible tragédie qui a pour seule issue, la perte de soi [9]. On qualifie bien souvent les personnes qui sont atteintes d'une DTA comme des êtres perdus [17]. Le désir de vouloir se protéger de sa propre déchéance et de protéger ses proches en leur évitant d'avoir à prendre soin d'une personne qui n'est qu'une partie de l'être qu'ils ont aimé est suffisant pour justifier des directives pouvant inclure une demande qui prendrait la forme d'un refus de traitement au sens large, d'un abandon et voire même d'une euthanasie. II y a un risque, comme le souligne Basson, que le malade rédige des DA qui nuiraient à son intérêt du simple fait qu'elles sont basées sur une vision partielle et possiblement faussement teintée de la réalité quotidienne de ces individus [7]. Soulignons le cas vécu de M. Hugo Claus, célèbre écrivain Belge, 
récipiendaire du prix Nobel de littérature qui, pour abréger la souffrance morale associée à l'annonce de son diagnostic d'Alzheimer, a demandé l'euthanasie et est mort à l'âge de 78 ans alors qu'il était encore en mesure de reconnaître ses proches, rire, parler et marcher [39].

La perspective d'un avenir imprévisible et estimé comme étant peu reluisant constitue un frein important à l'application contraignante des DA auprès des personnes qui sont atteintes d'une DTA dans le contexte où ces individus évoqueraient des DA en faveur d'une euthanasie. Nous sommes d'avis qu'il ne serait pas bienveillant d'encourager cette pratique qui risque d'émerger d'elle-même si les DA étaient considérées comme des absolus, voire des obligations. Nous sommes forcés de considérer que des dérives sont possibles lorsqu'on tente de promouvoir l'élaboration de DA chez les personnes atteintes d'une DTA. C'est pour ces raisons que les DA ne doivent pas être considérées comme des impératifs lorsqu'elles s'appliquent à la DTA $[23,40]$. Considérer les DA comme telles reviendrait à affirmer que la DTA est génératrice d'un quotidien infernal pour ces individus et qu'aucune qualité de vie n'est possible. Ceci va à l'encontre de notre expérience clinique de plus de 25 ans dans le domaine. Elle priverait la personne atteinte d'une DTA et ses proches d'espoir. L'émergence d'une qualité de vie insoupçonnée lui serait ainsi reniée. Rappelons-nous le paradoxe du handicap qui souligne que la qualité de vie dans une situation de handicap ne peut pas être correctement anticipée tant qu'elle n'est pas vécue [17]. Plus de la moitié des personnes qui vivent avec une situation de handicap rapportent avoir une bonne qualité de vie [17]. II est du devoir des professionnels de la santé qui gravitent autour de la personne atteinte de faire évoluer celle-ci ainsi que ses proches à ce sujet. C'est pourquoi nous sommes d'avis que l'élaboration et le déploiement des DA doivent être perçus comme un processus qui évoluera dans le temps et non comme un formulaire à remplir ou une simple conversation à tenir en début de maladie. Ce processus, pour demeurer bienfaisant, se doit d'être accompagné par des professionnels connaissant la maladie et des guides pratiques pour prévenir toutes dérives. II ne faut pas oublier qu'une personne souffrant d'une DTA peut perdre la capacité de faire un choix éclairé, mais elle demeure capable d'exprimer ses préférences (de manière possiblement différente) bien souvent jusqu'à la toute fin de la maladie. Elle conserve beaucoup plus longtemps qu'on ne le croit une autorité sur son bien-être tel que le sous-tend Jaworska [22]. Elle garde la capacité d'apprécier plusieurs dimensions de sa vie (manger, écouter de la musique, etc.) $[9,44]$.

L'inaptitude inhérente à la DTA est une notion qui a des implications légales importantes, elle a malheureusement comme effet pervers de faire oublier que la personne atteinte garde certaines compétences. Pour Appelbaum et Gzil, la compétence est "tâche-dépendante », elle s'évalue en fonction des capacités cognitives du malade, mais aussi en fonction de la nature et de la complexité de la décision qui est à prendre [22,30]. La personne qui souffre d'une DTA garde des capacités décisionnelles même si elle est inapte. Ce fait diffère de l'inaptitude du patient qui présente un coma ou une inconscience définitive comme cela est le cas du cancéreux en phase terminale. Pour pallier ce phénomène, Sokolowski introduit la notion d'autonomie contemporaine (autonomie résiduelle de la personne atteinte d'une DTA en temps réel) [9]. Elle souligne que « l'individu est bien plus que sa capacité à donner son consentement éclairé ". Nous sommes comme elle, d'avis que l'autonomie contemporaine de la personne atteinte d'une DTA doit être considérée au moment où des décisions s'imposent. Appliquer les souhaits antérieurs de la personne atteinte (qui est défini par Sokolowski comme son autonomie précédente) à sa réalité actuelle sans tenir compte de son autonomie contemporaine aurait un potentiel malfaisant dans le contexte d'une DTA que l'on doit absolument éviter [45]. Gzil qualifie d'attitude maltraitante le fait de disqualifier a priori toutes formes d'expression de la personne atteinte d'une DTA et de prendre des décisions sans la consulter [30]. Mme Lapierre devrait, par exemple, subir une chirurgie pour une cataracte et se voir possiblement installer un stimulateur cardiaque. Son neveu a accepté ces interventions pour le bien de sa tante tout en recherchant l'assentiment verbal de cette dernière. Que devraient faire son décideur de substitution et l'équipe de soins si, au moment des chirurgies, Mme Lapierre devient agitée et agressive de telle sorte qu'elle semble vivre l'expérience comme une agression? Sa façon de réagir peut possiblement indiquer qu'elle ne veut pas ces chirurgies où qu'elle n'a plus les capacités de comprendre pourquoi 
elle doit subir ces agressions corporelles qui lui sont imposées dans une visée bienfaisante. Peut-être que son comportement est sa façon à elle de nous dire qu'il faut réévaluer les coûts-bénéfices de ces interventions et possiblement revoir les stratégies d'intervention déployées pour favoriser une expérience adaptée à ses capacités [45]. II faut être en mesure de reconnaître la communication non verbale de ces personnes de manière à pouvoir qualifier leur confort [28,30]. Le rapport coûtsbénéfices des interventions dépend également du stade de la maladie et de la visée des soins (curative vs palliative) qui est mis de l'avant. L'acharnement thérapeutique occasionne des souffrances inutiles aux malades, à sa famille et aux soignants et des coûts inutiles au réseau de la santé [28]. Cependant, l'autonomie contemporaine ne peut pas être la seule autonomie considérée. Car laisser la personne qui souffre d'une DTA décider seule pour elle-même s'apparenterait à une forme d'abandon et de négligence considérant le fait que ses incapacités sont réelles et de plus en plus grandissantes au fur et à mesure que la DTA progresse [7]. Dans le cas d'une DTA, l'inaptitude ne justifie pas que l'autonomie décisionnelle actuelle de la personne ne compte plus [6]. Comme nous l'indiquent Hyed et Franklin, il ne faut oublier que: "It is perfectly true as philosophers say, that life must be understood backwards. But they forget the other proposition, that life must be lived forward » [46].

$\mathrm{Au}$ fait que les DA doivent être confrontées à l'autonomie contemporaine de la personne atteinte s'ajoute la perspective de sa qualité de vie et celle de son aidant. Wolff est d'avis que c'est la qualité de vie de la personne atteinte en temps réel qui devrait être la seule préoccupation du décideur de substitution au moment où il doit prendre une décision pour l'autre [17]. Selon lui, cette réalité dans le cas d'une DTA devrait avoir préséance sur les directives anticipées en faveur d'un refus de traitement ou d'une demande euthanasique. Le respect des DA est moralement louable dans une visée bienfaisante de permettre l'autodétermination de l'individu, mais elle peut devenir troublante et malfaisante si elle ne considère pas la qualité de vie actuelle de la personne au moment où ses volontés seront actualisées [17]. Comment pourrions-nous comme société honorer une demande euthanasique au moment où la personne atteinte bénéficie toujours d'une qualité de vie $[9,45]$ ? En effet, la douleur, la fatigue et le manque de contrôle sont les éléments clés en faveur d'une pauvre qualité de vie. Ces caractéristiques sont bien plus souvent associées à l'aidant qui est dans la plupart des cas à la fois le parent, le soignant et le décideur de substitution. Il est bien connu que les aidants éprouvent des "sentiments moraux très puissants et qu'ils s'interrogent en permanence sur le sens et la valeur de leurs actes » [30]. C'est pourquoi il devient pertinent selon nous d'ajouter la notion de qualité de vie de l'aidant qui doit être également considérée au moment de décisions importantes sans être pour autant la seule variable considérée [22]. Ce constat va dans le sens des recommandations de High [30].

La notion de qualité de vie est une notion difficile à saisir, c'est pourquoi un accompagnement par un intervenant de la santé sensible à la réalité de la personne atteinte est souhaitable (ex. : Société québécoise d'Alzheimer). C'est aux professionnels de la santé que revient le rôle de faire cheminer ces personnes et leurs aidants vers une approche de soin basée sur la qualité de vie partagée et renouvelée des êtres touchés par cette condition afin de permettre d'identifier et de moduler un nouveau sens à leur vie. Dans l'éventualité d'un constat d'absence de qualité de vie, qui demeure possible au stade terminal d'une DTA, il faut se demander si comme société nous serions prêts à honorer une demande euthanasique possiblement émise il y a de cela plusieurs années. Le cas ne sera pas possible au Québec puisque la LSFV exclut cette possibilité, mais nous estimons par contre que des pressions populaires risquent d'émerger sous peu comme ce fut le cas ailleurs $[1,34]$.

Malgré toutes les difficultés d'application que nous venons de soulever, nous partageons l'idée de Sokolowski, Widdershoven et Berghmans selon laquelle il est toujours préférable d'avoir une idée des volontés de la personne qui souffre d'une DTA, même si celles-ci sont sommaires et incomplètes $[9,45]$. Nous sommes d'avis qu'une décision prend tout son sens lorsqu'elle est en continuité avec les valeurs et l'histoire de la personne [16]. Nous estimons qu'il faut tenter d'instaurer cette pratique auprès de ce sous-groupe de personnes, car en l'absence de DA, la personne atteinte 
de la maladie d'Alzheimer, comme c'est le cas pour tout autre type de maladie, laisserait la règle de son meilleur intérêt être décidé par un tiers en tout temps [22]. Les tiers sont malheureusement trop souvent influencés par les équipes de soins et leurs propres intérêts. Et qu'en cas de dilemme et de divergences d'opinions, ces situations pourraient se voir soumis à l'éclairage des structures d'éthique clinique ou confier aux tribunaux [5,12]. II est possible de croire que sans directive, Mme Lapierre aurait possiblement quitté son domicile vers une ressource d'hébergement correspondant à ses besoins bien avant. Ces directives ont donné la force morale à son neveu et à son équipe de soins de mobiliser des ressources et des moyens supplémentaires pour honorer sa volonté. Pensons ici à l'implication de la voisine et au débranchement de sa cuisinière. Les directives anticipées ne sont certes pas toujours des absolus, mais elles méritent d'être reconnues lorsqu'elles se font l'expression de ceux qui aspirent à une vie digne [23,45]. " L'idéal étant d'avoir anticipé les complications les plus fréquentes et de les avoir abordées à l'avance avec la famille en expliquant les différentes attitudes possibles » [28]. N'avons-nous pas le devoir moral dans une société de droit de tenter de mettre tous les moyens possibles pour que le soi demeure souverain de sa destinée le plus longtemps possible ? Nous sommes d'avis qu'il faut seulement reconnaître que le soi dans le cas de la maladie d'Alzheimer n'arrête pas d'évoluer avec l'annonce du diagnostic et qu'il faut faire une place à l'autonomie contemporaine de la personne.

Nous souhaitons, en conclusion des arguments que nous venons de débattre, que les personnes nouvellement diagnostiquées de la maladie puissent émettre des DA (préférablement écrites devant minimalement deux témoins) si elles ont les capacités décisionnelles de le faire (le médecin devrait agir à titre de guide à ce niveau et devrait demander des investigations supplémentaires en cas de doute). Ces DA devraient contenir le choix d'un décideur de substitution (et possiblement des remplaçants en cas d'incapacité de celui-ci étant donné le caractère évolutif longitudinal de cette maladie), les préférences de la personne atteinte concernant les soins techniques et pharmacologiques normalement associés aux instants précédant la mort, mais aussi ceux associés au traitement (médication) et aux enjeux sociaux des soins qui ont un impact sur son bien-être (ex : lieu de vie et la contention), la participation à la recherche et toutes autres volontés pertinentes quant à son devenir (comme son consentement à des interventions chirurgicales mineures et le don d'organe). Le décideur de substitution devrait être informé de son mandat dès l'émission des DA de même que des volontés qu'elles contiennent dans le but de pouvoir discuter avec elle de toutes les dimensions qu'elles couvrent et tenter d'éliminer, autant que possible, les ambiguïtés et les oublis. Ces DA devraient être en tout temps annexées au dossier médical et consultées au moment où des décisions importantes doivent être prises. Nous sommes d'avis, à la lumière de tous les arguments dont nous venons de discuter, que les DA chez les individus atteints d'une DTA devraient être élaborées avec la perspective de chercher à garder le soi précédent souverain le plus longtemps possible pourvu qu'il aille de pair avec la qualité de vie de la personne au moment où elles sont déployées et qu'elles respectent autant que possible sa volonté en temps réel (autonomie contemporaine). Nous souhaitons que la qualité de vie du soignant, qui est souvent à la fois un proche parent et son décideur de substitution, soit également évoquée dans une visée de moindre mal, car la pérennité de son implication est directement reliée au bien-être de la personne atteinte. La prise de décision pour l'être aimé atteint d'une DTA réside dans la capacité du décideur de substitution de se suppléer à l'autre en tentant d'honorer son être et son parcours, tel qu'il fut avant sa maladie, avec ses croyances et ses valeurs tout en respectant l'être actuel qu'il est devenu et en ne perdant pas de vue sa qualité de vie ainsi que la sienne. Ces recommandations vont de pair avec l'association Alzheimer Europe [47]. Ce mandat représente un défi de taille pour le décideur de substitution. Un accompagnement professionnel et des repères normatifs pour assurer la visée bienfaisante de l'élaboration et le déploiement des DA nous apparaissent essentiels pour les personnes atteintes d'une DTA qui désirent se prononcer en amont de leur incapacité décisionnelle. II en va de même pour le décideur de substitution qui aura la responsabilité de les actualiser et les professionnels de la santé qui auront la tâche de les accompagner. 
La présente analyse vient démontrer la pertinence de développer chez les personnes souffrant d'une DTA une approche clinique complémentaire aux politiques publiques déjà existantes et en voie d'installation pour assurer une utilisation bienfaisante du concept éthique des DA. Cette affirmation est en accord avec les recommandations de Singer qui en 1996 venait promouvoir le développement de « specific-disease-approach » [8] pour ce concept. Car pour les personnes souffrant d'une DTA, les DA doivent aussi s'inscrire dans une visée de qualité de vie et non seulement de fin de vie. Tout comme Hirsch, nous estimons que l'individu évolue au fil des expériences qu'il vit et que le respect absolu d'une décision prise avant même d'avoir vécu cette expérience s'avère dangereux [23]. II est difficile d'anticiper le parcours que fera vivre une DTA à la personne. II est utopique de croire que l'application du concept des DA pour les personnes souffrant d'une DTA sera l'histoire d'une simple conversation ou de quelques mots sur une feuille de papier alors que cette condition évolue sur plusieurs années [8]. S'en remettre uniquement à des dispositifs administratifs serait tout simplement dangereux et peu satisfaisant pour tous [12]. "Perhaps the advance directive's greatest virtue of all is the role it is playing in motivating healthcare professionals to embrace the intention of honouring the demented person's wishes, interest and values, but to do so through engagement in advance care planning with their patients as an ongoing process and in a proactive way » [9].

Cette visée de Sokolowski est en accord avec Widdershoven et Berghmans qui stipulent que les DA doivent être plutôt perçues et utilisées comme un outil de communication qui donne du sens à la vie " a process of meaning making » [45]. L'émission et le déploiement de DA chez les individus atteints d'une DTA doivent être présentés et articulés comme un processus modulable de revalidation des ententes qui tient compte de l'autonomie précédente et contemporaine de la personne atteinte, sa qualité de vie et celle de son soignant. Cette visée plus proche de la définition de Singer doit être accompagnée par des repères normatifs qui demeurent à développer.

\section{Conclusion}

La promotion de l'élaboration et le déploiement de directives anticipées chez les personnes nouvellement diagnostiquées d'une DTA aux stades précoces sont pertinents et bienfaisants étant donné l'atteinte progressive et prévisible des capacités décisionnelles de la personne atteinte. Le respect de l'autodétermination de ces individus est au cœur des valeurs de notre société de droit et au centre de tous les codes de déontologie des professionnels de la santé au Québec. Les directives anticipées permettent à la norme du consentement éclairé de s'actualiser au moment où le malade perd ses capacités décisionnelles. Cette notion comporte selon nos observations des flous conceptuels et méthodologiques qui contribuent entre autres au fait que ce moyen est peu reconnu et utilisé. Au Québec, le Code civil indique au décideur de substitution qu'il devrait s'en tenir aux volontés que la personne inapte a pu manifester et la loi reconnaît au majeur apte le droit de choisir en amont de son inaptitude son représentant (mandat en cas d'inaptitude). Le régime des DMA associé à la LSFV permet l'expression, toujours en amont de l'inaptitude, des volontés anticipées du majeur pour certains soins précédant la mort et leur donne une valeur contraignante.

L'histoire de Mme Lapierre nous a permis de souligner toute la complexité de l'utilisation clinique du concept éthique de DA dans le contexte de la DTA. Elle nous a permis de constater que ces directives ne peuvent être considérées comme des absolus étant donné les spécificités associées à cette maladie dégénérative (atteinte progressive des capacités décisionnelles évoluant sur plusieurs années). Notre réflexion a été élaborée en fonction des défis particuliers que vivent les personnes souffrant d'une DTA (atteinte des capacités décisionnelles en présence d'une anosognosie). La généralisation de notre propos aux autres formes de démence n'est pas indiquée.

Pour assurer la visée bienfaisante du concept éthique de DA pour les personnes atteintes d'une DTA, nous énonçons :

1. Le besoin d'élargir la notion de directives anticipées aux soins en cours de maladie (incluant le choix d'un lieu de vie) et non seulement aux soins de fin de vie ; 
2. la pertinence d'assurer un accompagnement par un professionnel de la santé, par un organisme sans but lucratif sensible à la réalité de ces personnes ou par des guides pratiques afin de permettre que ces directives soient le plus complètes et réalistes et que leur déploiement soit bienfaisant, et

3. la nécessité d'actualiser ce concept comme un processus (approche graduelle qui permet de refaçonner les ententes) où les DA se modulent au jugement du décideur de substitution qui doit tenir compte de la volonté de la personne atteinte en temps réel (autonomie contemporaine), sa qualité de vie et celle de son soignant.

Nous sommes d'avis que c'est au décideur de substitution et aux professionnels de la santé qu'appartiennent le rôle de donner un sens renouvelé aux DA de ces personnes pour assurer que l'orientation des soins soit empreinte de sens pour la personne atteinte, ses proches et la société.

Nous avons dénoncé l'absence de politiques de santé en ce sens au Québec pour les personnes atteintes d'une DTA ainsi que le fait que le développement de la reconnaissance des directives anticipées soit associé à la Loi concernant les soins de fin de vie. Nous déplorons que les notions rattachées aux DA soient développées exclusivement au Québec en fonction des besoins des malades qui souffrent d'une incapacité décisionnelle de courte durée et précédant la mort. Nous avons défendu l'idée que l'émission et le déploiement du concept éthique de DA requièrent un accompagnement répondant aux besoins spécifiques inhérents à la DTA et qu'elles ne doivent jamais être la seule option considérée pour ces malades [10].

II faut maintenant sensibiliser les principaux acteurs de la société incluant les personnes atteintes et leurs proches à ces faits pour éviter plusieurs dérives et prévenir que des situations comme celle de Mme Lapierre se répètent. II faut prôner un changement de paradigme au niveau de l'approche qui doit être perçue pour ces personnes comme un processus de revalidation des ententes plutôt qu'une seule conversation ou qu'un seul formulaire à remplir. Le développement de politiques de santé incluant des repères normatifs et des guides de pratique qui tiendront compte de notre analyse nous apparaît essentiel pour assurer une vie et une fin de vie digne à ces malades. II apparaît maintenant urgent comme le souligne Basson de réfléchir aux modalités de mise en place de politiques pertinentes et de soutenir en ce sens les organismes qui ont à cœur le bien-être de ces malades pour favoriser le développement de celles-ci [7]. Comme le souligne Singer, plus il existera de programmes pour encourager l'application en clinique des DA, plus elles seront utilisées [8]. Nous espérons que le présent texte sera, à sa façon, un élément déclencheur en ce sens.

\section{Biographie}

Line Perreault est ergothérapeute dans le domaine de la gériatrie et la psychogériatrie depuis plus de 26 ans. Elle a travaillé en centre hospitalier universitaire (unité de courte durée gériatrique et clinique gériatrique externe, Hôpital Notre-Dame du CHUM) et dans la communauté (CLSC, CSSS LucilleTeasdale). Elle fut membre du comité d'éthique clinique du CSSS Lucille-Teasdale de 2006 à 2015 et présidente de 2011 à 2014. Elle est nouvellement détentrice d'une maîtrise en bioéthique de l'Université de Montréal.

\section{Références}

1. Fewing R, Kirk TW, Meisel A. A fading decision. The Hastings Center Report. 2014;44(3):14-6.

2. Éducaloi. La procuration (ou le mandat). Montréal (Qc): Éducaloi; 2015.

3. Gouverment du Québec. Directives médicales anticipées. Portail santé mieux-être. 2015.

4. Bergman H. Relever le défi de la maladie d'Alzheimer et des maladies apparentées: une vision centrée sur la personne, l'humanisme et l'excellence. Rapport du comité d'experts en vue de l'élaboration d'un plan d'action pour la maladie d'Alzheimer. Mai 2009. 
5. Beauchamp T, Childress J. Le respect de l'autonomie. Dans: Les principes de l'éthique Biomédicale. Paris : Les Belles Lettres. 2008. p. 91-166.

6. Rich B. Advance directives: The next generation. Journal of Legal Medicine. 1998;19(1):63-97.

7. Basson M, Burgeron P-E, Herson S. Prise de décision, consentement et directives anticipées: approches éthiques, Projet européen ALCOVE (ALzheimer COoperative Valuation in Europ), Workpackage 7 : Rights, autonomy and dignity of people with dementia. Espace National de réflexion éthique sur la maladie d'Alzheimer. Janvier 2011.

8. Singer PA, Robertson G, Roy DJ. Bioethics for clinicians: 6. Advance care planning. CMAJ. 1996;155(12):1689-92.

9. Sokolowski M. Why use of advance directives with the dementia population is problematic. The JCB Voice. 2011;16(10):13-15.

10. Besirevic V. End-of-life care in the 21st century: advance directives in universal rights discourse. Bioethics. 2010;24(3):105-12.

11. Hottois G, Missal JN. Nouvelle encyclopédie de bioéthique. Bruxelles: De Boeck Université; 2001.

12. Philips-Nootens S. La personne en fin de vie: Le regard du droit civil du Québec. Revue de droit de l'Université de Sherbrooke. 2009;40(1-2):327-64.

13. Fournier V, Berthiau D, Kempf E, d'Haussy J. Quelle utilité des directives anticipées pour les médecins? La Presse Médicale. 2013;42(6):e159-e69.

14. Teixeira A, Hanvey L, Tayler C, Barwich D, Baxter S, Heyland D. What do Canadians think of advanced care planning? Findings from an online opinion poll. BMJ Supportive \& Palliative Care. 2013. doi:10.1136/bmjspcare-2013-000473

15. Epstein AS, Volandes AE, O'Reilly EM. Building on individual, state, and federal initiatives for advance care planning, an integral component of palliative and end-of-life cancer care. Journal of Oncology Practice. 2011;7(6):355-9.

16. Wasylenko E. Advance care planning and goals of care designations- a changing focus and altered infrastructure. The JCB Voice. 2010;16(2):8-9.

17. Wolff J. Dementia, death and advance directives. Health Econ Policy and Law. 2012;7(4):499506.

18. Philips-Nootens S. L'hébergement forcé: quels intérêts, quels enjeux? - Regards sur la jurisprudence récente... et plus. Dans : Barreau du Québec. La protection des personnes vulnérables 2009, volume 301. Québec : Éditions Yvon Blais; 2009.

19. Philipsen NC, Haynes DR. The similarities between birth plans and living wills. The Journal of Perinatal Education. 2005;14(4):46-8.

20. Kerrigan S, Ormerod I. Advance planning in end-of-life care: legal and ethical considerations for neurologists. Prat Neurol. 2010;10(3):140-4.

21. Heyland DK, Barwich D, Pichora D, Dodek P, Lamontagne F, You JJ, et al. Failure to engage hospitalized elderly patients and their families in advance care planning. JAMA Internal Medicine. 2013;173(9):778-87.

22. Gzil F. Alzheimer: questions éthiques et juridiques. Gérontologie et société. 2009;1(128129):41-55.

23. Hirsch E. Fin de vie: les véritables enjeux des directives anticipées. 10 février 2014.

24. Société Alzheimer de Québec. Statistique. 2015.

25. Collette $C$. Service spécialisés destinés aux personnes souffrant de la maladie d'Alzheimer. ETMIS, octobre 2012;8(16).

26. Arcand M, Hébert R. Maladie d'Azheimer. Dans: Précis pratique de gériatrie. $3^{\mathrm{e}}$ éd. Acton Vale(QC): Edisem; 2007. p. 257-67.

27. Hirsh $\mathrm{E}$. Alzheimer : Engagements éthique et soin. Espace national de réflexion éthique sur la maladie d'Alzheimer, 2009.

28. Lopez-Tourres F, Lefebvre-Chapiro S, Fétéanu D, Trivalle C. Soins palliatifs et maladie d'Alzheimer. La revue de médecine interne. 2009;30:501-7.

29. Mak E, Chin R, Ng L, Yeo D, Hameed.S. Clinical associations of anosognosia in mild cognitive impairment and Alzheimer's disease. Int J Geriatr Psychiatry. 2015;30(12):1207-14. 
30. Gzil F. Le souci des autres: enjeux éthiques de l'accompagnement informel des malades d'Alzheimer. NPG Neurologie-Psychiatrie-Gériatrie. 2008;48(8):8-21.

31. Société Alzheimer Montréal. Communication personnelle avec L. Perreault L. novembre, 2014.

32. Deleury E, Goubau D. Le droit à l'intégrité physique. Le droit des personnes physiques, $5^{e}$ ed. Québec: Éditions Yvon Blais; 2014.

33. Gouvernement du Québec. Code civil du Québec, 1991(2015), C64.

34. Alvargonzalez D. Alzheimer's disease and euthanasia. Journal of Aging Studies. 2012;26(4):377-85.

35. Andorno R, Comité directeur pour la bioéthique. Les souhaits précédemment exprimés au sujet des soins de santé Principes communs et différentes règles applicables dans les systèmes juridiques nationaux. Rapport au Conseil de l'Europe. 35ème réunion 2-5 décembre, 2008.

36. Rabia L. Nouvelles directives anticipées de la FMH et de l'ASSM. Bulletin des Médecins Suisses. 2011;92(23/24):873.

37. Académie Suisse des Sciences Médicales. Directives anticipées, directives Médico-Éthiques. 1 janvier 2013.

38. Groupe thématique « Directives Anticipées » de l'Espace Éthique Rhône-Alpes. Les directives anticipées en pratique, guide conseil à l'usage des professionnels. 5 octobre 2012.

39. BBC News. Author Claus dies by euthanasia: 21 mars 2008.

40. Bennett D, Yu L, Yang J, Srivastava G, Aubin C, De Jager P. Epigenomics of Alzheimer's disease. Translational Research. 2015;165(1):200-20.

41. Lavoie P, Guberman N, Marier P. La responsabilité des soins aux aînés au Québec: du secteur public au privé. Etude IRPP no 48, septembre 2014.

42. Coalition canadienne des organismes de bienfaisance en santé. Énoncé de principes sur l'accès aux soins à domicile. 22 septembre 2011.

43. CROP. Sondage auprès des Québécois âgés de 50-64 ans sur le vieillissement, Demandé par l'AQESSS Montréal: CROP; 2010.

44. Duzan B, Fouassier P. Maladie d'Alzheimer et fin de vie: aspects évolutifs et stratégies thérapeutiques. Médecine palliative - Soins de support - Accompagnement - Éthique. 2011(10):230-41.

45. Widdershoven G, Berghmans R. Advance directives in dementia care: from instructions to instruments. Patient Education and Counseling. 2001;44(2):179-86.

46. Heyd D, Franklin GM. Life plans: do they give meaning to our lives. The Monist. 2010;93(1):17-37.

47. Alzheimer Europe. Position paper on the use of advance directives, 6 août 2009. 